\title{
Multiobjective analysis for the design and control of an electromagnetic valve actuator
}

\author{
P Stewart $^{1 *}$, D Gladwin ${ }^{1}$, and P J Fleming ${ }^{2}$ \\ ${ }^{1}$ Department of Electronic and Electrical Engineering, University of Sheffield, Sheffield, UK \\ ${ }^{2}$ Department of Automatic Control and Systems Engineering, University of Sheffield, Sheffield, UK
}

The manuscript was received on 4 September 2006 and was accepted after revision for publication on 24 January 2007.

DOI: 10.1243/09544070JAUTO439

\begin{abstract}
The electromagnetic valve actuator can deliver much improved fuel efficiency and reduced emissions in spark ignition (SI) engines owing to the potential for variable valve timing when compared with cam-operated, or conventional, variable valve strategies. The possibility exists to reduce pumping losses by throttle-free operation, along with closed-valve engine braking. However, further development is required to make the technology suitable for acceptance into the mass production market. This paper investigates the application of multiobjective optimization techniques to the conflicting objective functions inherent in the operation of such a device. The techniques are utilized to derive the optimal force-displacement characteristic for the solenoid actuator, along with its controllability and dynamic/steady state performance.
\end{abstract}

Keywords: solenoid, multiobjective design, optimization, controllability, automotive, electromagnetic valve actuator

\section{INTRODUCTION}

Actuator controllability is often considered relatively late in the process of project development. The total mobile mass of the valve, collets, and spring is often predefined, along with the spring rate and preload. The most commonly used valve for this application is the poppet valve which has the most desirable characteristic combination of gas flow properties, closed sealing, ease of lubrication, and heat transfer. Subsequent selection of actuator hardware to achieve the defined performance criteria is then often made in terms of static force characteristics, after which time the control developer must design algorithms to achieve the desired real-time dynamic performance. In this paper a structured approach to actuator selection and control system design is presented, focusing on the control of normal-force solenoid actuators in the context of strict performance criteria, although it will be shown that the technique can form the basis of a more generic approach. The aim of the multiobjective design approach presented in

* Corresponding author: Department of Electronic and Electrical Engineering, University of Sheffield, Mappin Street, Sheffield S1 3JD, UK. email: p.stewart@shef.ac.uk this paper is to select the actuator in such a way that the control engineer implements the algorithms on a platform that can achieve the dynamic performance required by the design specifications while staying within other design implementation bounds such as current and voltage limits. The approach is as follows. First, a suitable candidate force-displacement profile that would allow the mechanical system to comply with the performance criteria will be found using multiobjective evolutionary search techniques. This makes available to the electromagnetic design engineer a far more detailed force profile than simply providing a maximum force requirement. This approach does not consider or include the dynamic limitations imposed by the behaviour of current, flux, and force in the actuator. A candidate electromechanical actuator will thus be examined by a similar multiobjective approach, this time to confirm its controllability in the context of the project dynamic performance criteria. Finally, a gain-scheduled position-velocity controller will be designed, again using multiobjective techniques. It will be shown that the application of multiobjective techniques can contribute to the design of control systems that are controllable in the sense of extremely complex and in some cases conflicting design criteria. 


\section{PROBLEM DEFINITION}

Since it is freed from a fixed geometric relationship with the crankshaft owing to its independent control, the EMV offers great potential improvements in engine performance $[\mathbf{1}]$. The standard configuration for an electromagnetic valve actuator (EMV) consists of a spring and two electromagnets held within a rigid casing which acts directly on the stem of a standard poppet valve (Fig. 1).

The EMV configuration has balanced opposing springs (to minimize the reactive power driven through the power electronics) of equal rate, a full stroke of $8 \mathrm{~mm}$, in equilibrium position at $4 \mathrm{~mm}$, and opposing solenoids to effect control over the movement of the armature which bears on the valve stem. The two springs exchange potential energy during the motion of the valve between the two operating points (fully open and fully closed). The actuators compensate for the energy losses during motion, and also fulfil the requirement to hold the valve at the operating points. The controllability of the system is evaluated in the context of the project performance requirements [2].

1. The transition time from fully open to fully closed must be less than $3 \mathrm{~ms}$.

2. To minimize acoustic noise, landing velocity to the fully closed position must be less than $0.05 \mathrm{~m} / \mathrm{s}$.

3. To minimize acoustic noise, the closing of the valve clearance (the clearance between the armature and the valve stem to ensure positive closing, otherwise known as lash) from the fully open position must be less than $0.05 \mathrm{~m} / \mathrm{s}$.
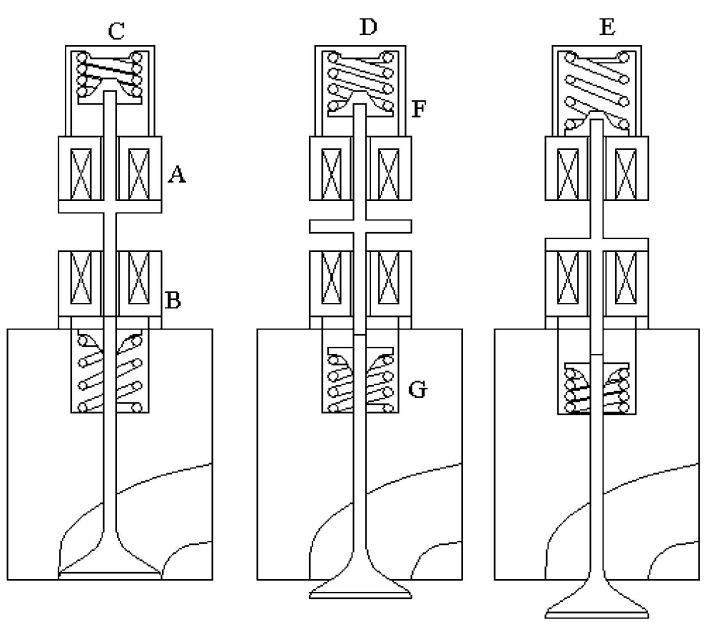

Fig. 1 Electronically controlled engine valve actuator: A, upper solenoid; B, lower solenoid; C, fully closed; D, equilibrium position; E, fully open; F, upper spring; $G$, lower spring
4. To minimize acoustic noise, landing velocity to the fully open position must be less than $0.05 \mathrm{~m} / \mathrm{s}$.

5 . At a supply voltage of $42 \mathrm{~V}$, the maximum catching current must typically be $40 \mathrm{~A}$.

6. At a supply voltage of $42 \mathrm{~V}$, the maximum holding current must typically be $7.5 \mathrm{~A}$.

The performance definition given above is the base formulation of an objective function for the mechanical system which will be extended to include a subobjective function for the electrical operation of the electrical system. The designed control algorithm must be able dynamically to open and close the valve subject to these operational constraints. It can be seen that a set of position-velocity trajectories exist to satisfy these requirements, but the choice of actuator to achieve this performance is an extremely complex one, compounded by the inherently non-linear force capabilities of solenoid actuators. Although controllability analysis has been applied to non-linear systems [3-5], a point is reached where the performance requirements of the system, in conjunction with all the other lumpedsystem non-linearities and discontinuities, become too difficult a task for standard mathematical analysis. Evolutionary algorithms have been applied to a variety of control system design problems [6, 7]. More specifically, genetic algorithms have been used [8] to optimize the two-dimensional finite element electromagnetic design of normal-force solenoid actuators. This was, however, a single-objective approach to optimize the force-displacement profile over a range of air gaps. In the approach under consideration here, the design will proceed as follows. The dynamic force-displacement characteristic required of the mechanical system will be analysed, an actuator topology will be selected, and finally the actuator controllability will be confirmed. An experimentally verified mechanical model in Simulink is used for performance verification, and also as the platform for the multiobjective searches.

\section{FORCE-DISPLACEMENT OPTIMIZATION}

The EMV system described in this paper was developed as part of the EU Framework V project ELVAS (Electronic Valve Actuation Systems) which defined the physical parameters of the mechanical system as follows:
(a) total mobile mass $90 \mathrm{~g}$;
(b) spring rate $75 \mathrm{~N} / \mathrm{mm}$;
(c) friction constant $13 \mathrm{~N} / \mathrm{m} \mathrm{s}$;
(d) spring preload $150 \mathrm{~N}$. 
The mechanical valve system can be modelled (Fig. 2) as [2]

$$
\ddot{y}=\frac{F_{1}-F_{2}-B \dot{y}-2 K y}{m}
$$

where $y$ is the position of the armature relative to the equilibrium position, $F_{1}$ and $F_{2}$ are the lower and upper actuator forces respectively, $B$ is the friction constant, $K$ is the effective spring rate of the pair of springs, and $m$ is the effective total mobile mass. For the purposes of analysis, the transition from valve fully open to valve fully closed will be considered, and an objective function to be assessed by the multiobjective genetic algorithm (MOGA) will be formulated. The objective function is defined as follows

$$
\begin{aligned}
f(y, \dot{y})=\min \{ & t_{\mathrm{r}}^{1}+\left(\int_{\mathrm{r}}^{1} F_{\mathrm{b}}^{\mathrm{t}} \mathrm{d} t\right)\left(F x^{2}\right)_{\mathrm{b}}^{\mathrm{t}} \\
& +[F(\max )]_{\mathrm{b}}^{\mathrm{t}}\left(\dot{y}_{1}\right)_{\left(\mathrm{lim} \leqslant 0.05 \mathrm{~ms}^{-1}\right)} \\
& \left.+\left(\dot{y}_{\mathrm{vg}}\right)_{\left(\mathrm{lim} \leqslant 0.05 \mathrm{~ms}^{-1}\right)}+\left[\frac{\mathrm{d} F}{\mathrm{~d} t}(\max )_{\mathrm{b}}^{\mathrm{t}}\right]\right\}
\end{aligned}
$$

where the objectives are articulated as follows.

Objective 1. Minimization of the transition time from release to landing, $t_{\mathrm{r}}^{1}$.

Objective 2. Minimization of the integral of force with respect to time from release to landing with respect to the bottom and top actuators, $F_{\mathrm{b}}^{\mathrm{t}}$.

Objective 3. Minimize the applied force at large air gaps, where $x$ is the distance from the active actuator.

Objective 4. Minimize the maximum applied force value.

Objective 5. The landing speed must be less than $0.05 \mathrm{~m} / \mathrm{s}$, where $\dot{y}_{1}$ is the landing velocity.
Objective 6. On release, the maximum armature velocity closing the valve gap must be less than $0.05 \mathrm{~m} / \mathrm{s}$, where $\dot{y}_{\mathrm{vg}}$ is the contact velocity of the armature on the valve stem.

Objective 7. Minimize the integral of force derivative with respect to time.

Objectives 1, 5, and 6 relate directly to stated design criteria, but the other objectives require some clarification. Objective 2 seeks to minimize the overall power consumption of the actuators, since the force developed by the actuators will be related to current, with related $i^{2} r$ copper losses in the windings. Objective 3 reflects the typical solenoid force-displacement characteristic [9] which can be approximated as

$$
F=\frac{K_{\mathrm{f}}(i)^{2}}{e^{2}}
$$

where $K_{\mathrm{f}}$ is the solenoid force constant, $i$ is the current, and $e$ is the air gap. Consequently, solutions that require force at large air gaps are heavily penalized. Objective 4 seeks to minimize the overall maximum value of the applied force, again to reduce the effects of losses. Finally, objective 7 seeks to find the smoothest force profile in order to maximize the potential for controllability of the actuators, since, owing to high reluctance, force derivatives at small air gaps are a severe limiting factor to the dynamic performance of solenoid actuators. The genetic algorithms toolbox for Matlab with the multiobjective extension tools (MOGA) developed at the University of Sheffield was utilized to perform the simulation routines.

\subsection{MOGA overview}

Formally, and without loss of generality [10], multiobjective optimization can be expressed as: minimize $\boldsymbol{f}(\boldsymbol{x})$, where $\boldsymbol{f}(\boldsymbol{x})=\left[f_{1}(\boldsymbol{x}) \ldots f_{n}(\boldsymbol{x})\right]$ is a vector of

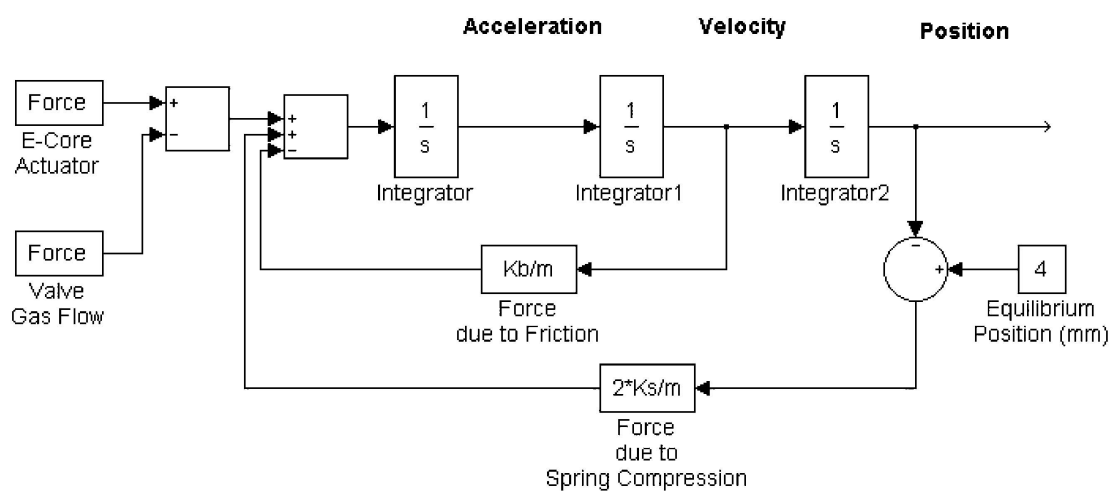

Fig. 2 Mechanical model 
objective functions, $n$ is the number of objectives or criteria to be considered, $\boldsymbol{x}=\left(x_{1} \ldots x_{p}\right)$ is a vector of decision variables, and $p$ is the number of decision variables that comprise the complete solution. In the absence of preference information, solutions to multiobjective problems are compared using the notion of Pareto dominance. A particular solution $\boldsymbol{x}$, with associated performance vector $\boldsymbol{u}$, is said to dominate another solution $y$ with performance vector $\boldsymbol{v}(\boldsymbol{x}<\boldsymbol{y})$ if the former performs at least as well as the latter across all objectives, and exhibits superior performance in at least one objective. A solution is said to be Pareto optimal if it is not dominated by any other possible solution. The Pareto front is the set of points in criterion space that correspond to the Pareto optimal solutions. Without a priori or progressive preference articulation, a multiobjective search engine will generally aim to discover a family of solutions that provide a good representation of the Pareto front (Figs 3 and 4).

The first Pareto-based multiobjective evolutionary algorithm (MOEA) to be published was the multiobjective genetic algorithm (MOGA) [11]. Genetic algorithms are suitable search engines for multiobjective problems primarily because of their population-based approach. An MOEA is capable of supporting diverse, simultaneous solutions in the search environment. A carefully designed GA is robust in the face of ill-behaved cost landscapes featuring attributes such as multimodality and discontinuity. Furthermore, the GA methodology offers a flexible choice of decision variables and objective

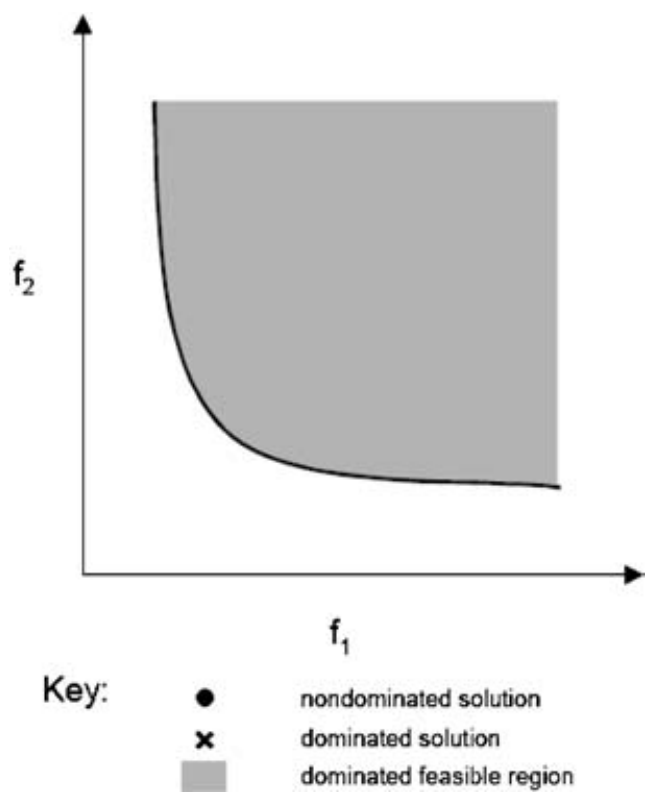

Fig. 3 Non-dominated solution

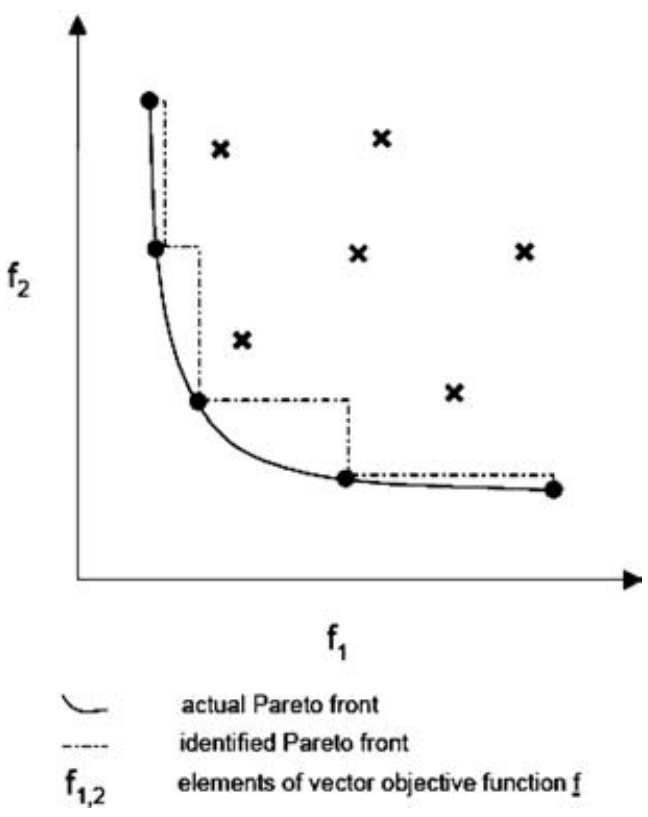

Fig. 4 Pareto optimality

specifications. A general schematic of the MOGA is shown in Fig. 5. The MOGA framework can be seen to incorporate all the elements of the standard, single-objective, genetic algorithm. A population of potential solutions is instantiated, then assessed and manipulated over a number of iterations in order to obtain a good solution or set of solutions. Performance assessment, selection, genetic operators (such as crossover and mutation), and reinsertion phases are functionally, in a general sense, the same for the MOGA as for the standard GA. Population distribution analysis, in which a measure of the density of the population is made, has also been applied in the single-objective case to cater for multimodal cost landscapes. The results of this analysis are used in niching and mating restriction schemes. Multiobjective ranking, which impacts primarily on fitness assignment, is the key difference between the MOGA and a standard GA. Interaction with a decision-maker (DM), or group of decision-makers, is made explicit in Fig. 5. The DM may choose to introduce $a$ priori information into the initial population (at the very least, this would include appropriate limits on decision variables), as is sometimes the case in standard GA applications. With the MOGA, the DM can also seek to influence the search while it is in progress by expressing preference for particular solutions or, more generally, the likely attributes of a good solution. The essential difference between a MOGA and a single-objective GA is the method by which fitness is assigned to potential solutions. Each solution will have a vector describing 


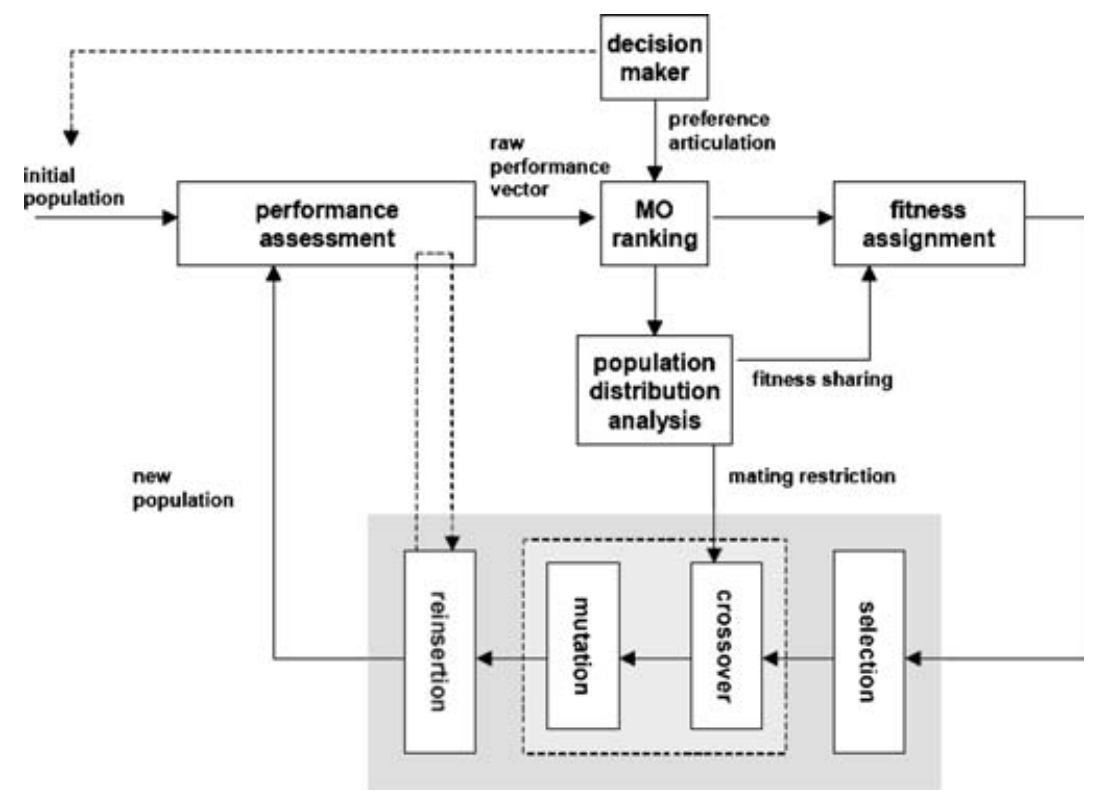

Fig. 5 MOGA schematic

its performance across the set of criteria. This vector must be transformed into a scalar fitness value for the purposes of the GA. This process is achieved by ranking the population of solutions relative to each other, and then assigning fitness based on rank. Individual solutions are compared in terms of Pareto dominance. This notion was introduced into the field of genetic algorithms by Goldberg [12]. MOGA uses a variation of Goldberg's proposition in order to determine ranks. Each individual is assigned a rank based on the number of individuals by which it is dominated (Fig. 6). In the absence of preference information, Pareto dominance is used to discriminate between two competing solutions. However, by involving a DM in the search, other factors can be used to determine superiority. Fonseca and Fleming [7] introduced a preferability operator, which discriminates between solutions on the basis of which is preferred by the DM. In the Fonseca and Fleming scheme, the DM can set goal levels and

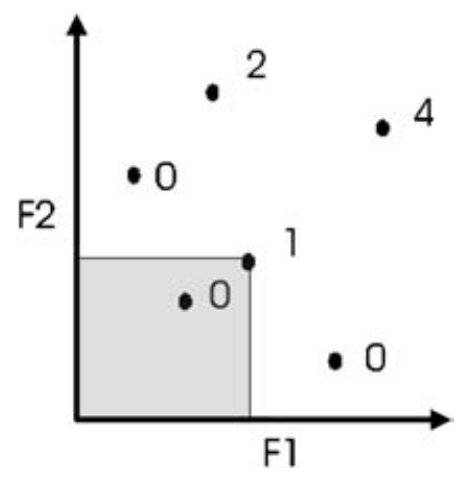

Fig. 6 Multiobjective ranking priorities for each of the objectives. These can be refined as the search progresses. This information feeds into the preferability operator, which is used to rank solutions in a similar fashion to the standard Pareto-based approach. Each potential solution is given a rank based on how many other solutions are preferred to it. The preferability operator can be seen as a unification of several popular preference articulation schemes adopted in the wider operational research community. Pareto optimality, the lexicographic method, goal programming, constraint satisfaction, and constrained optimization can all be described by special cases of the preferability operator.

\subsection{Moga application}

MOGAs [11] have routinely been applied to control optimization problems such as gain scheduling or controller parameter optimization $[\mathbf{1 3}, \mathbf{1 4}]$. However, in the present case an attempt is being made to identify the optimal dynamic force-displacement characteristic to operate within the bounds set by the design criteria. The decision variables are in this case assigned to a quantized map of the position of the armature, with variables clustered more closely at small air gaps, since the bulk of the control action is anticipated to occur at air gaps less than $1 \mathrm{~mm}$, along with the soft valve gap closure after $0.3 \mathrm{~mm}$. A 27-variable objective function was implemented, representing $1 \mathrm{~mm}$ steps from release to landing, with $0.1 \mathrm{~mm}$ steps within $1 \mathrm{~mm}$ of each actuator. The parameters associated with the MOGA set-up are given in the Appendix. Applying the multiobjective 
search algorithm to the mechanical system results in a velocity-position profile that satisfies the project design requirements (Figs 7 and 8).

The motion of the armature under the candidate force-displacement profile (Fig. 9) satisfies all the soft release, soft landing, and transition time criteria from the project definition. It also inherently possesses a relatively smooth force trajectory, which should also represent one of the lowest power consumptions when followed by a pair of actuators. This analysis considerably increases the detail of the design requirements that can be supplied to the electromagnetic design engineer. However, a limiting factor in the operation of solenoids at relatively small air gaps is due to the current and flux dynamics. An actuator candidate will now be considered to assess its performance suitability to achieve the required dynamics, using information derived from finite element analysis.

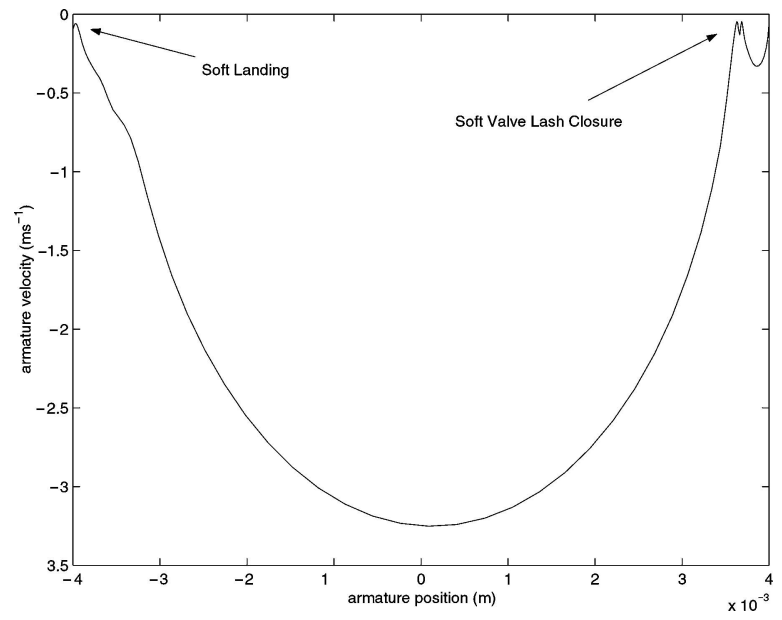

Fig. 7 Optimised position-velocity profile

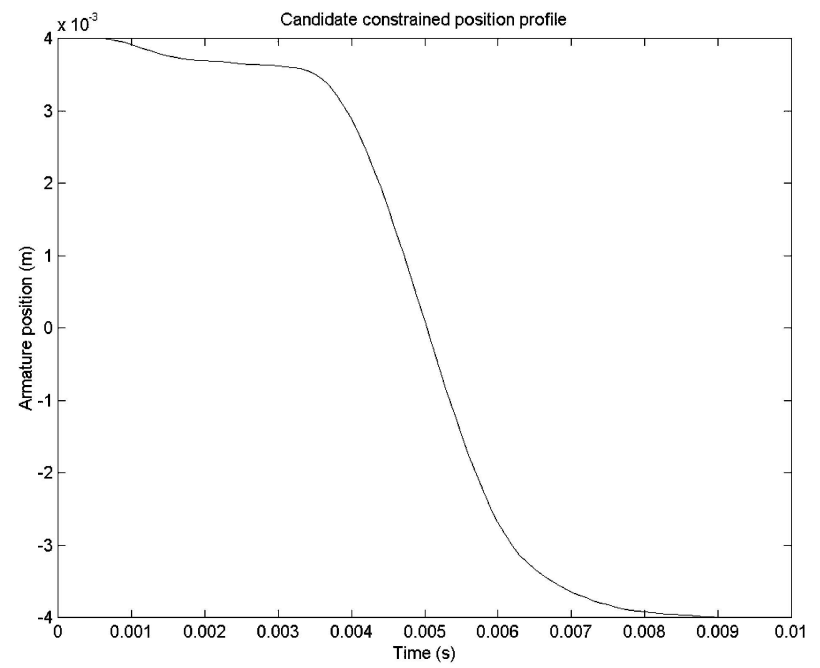

Fig. 8 Optimised position-time profile

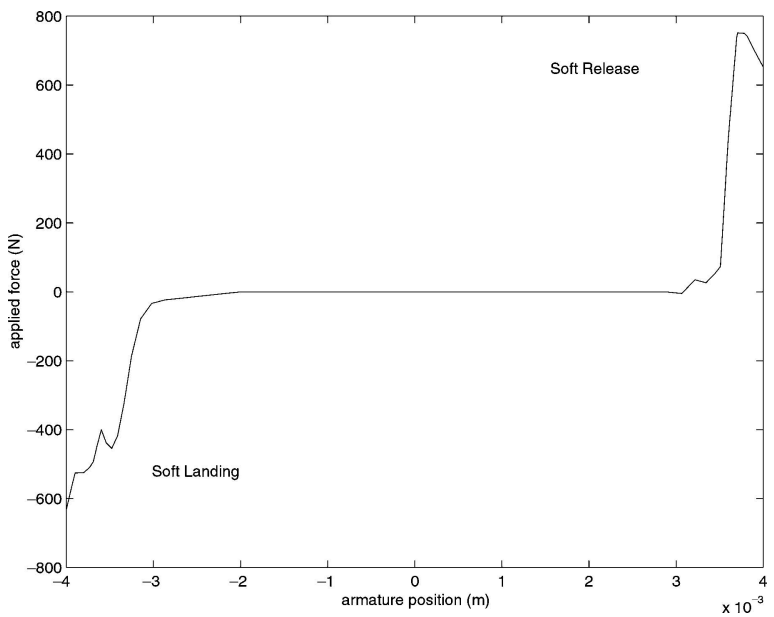

Fig. 9 Candidate force-displacement profile

\section{ACTUATOR MULTIOBJECTIVE CONTROLLABILITY ANALYSIS}

Assuming a given valve pitch for the particular cylinder head under consideration ( $33 \mathrm{~mm}$ ), a solenoid actuator can be designed and simulated using finite element methods $[\mathbf{1 5}, \mathbf{1 6}]$ based around a core size bounded by the valve pitch size, current and voltage limitations, and other dimensional limitations of the cylinder head. By way of example, applying a magnetostatic approach (force analysis at fixed air gaps), a force-current-displacement map for a particular design can be derived (Fig. 10). Comparison with the optimized force-displacement requirement (Fig. 9) confirms the suitability of the candidate actuator in steady state terms (Figs 11 and 12), but the controllability of the actuator in dynamic,

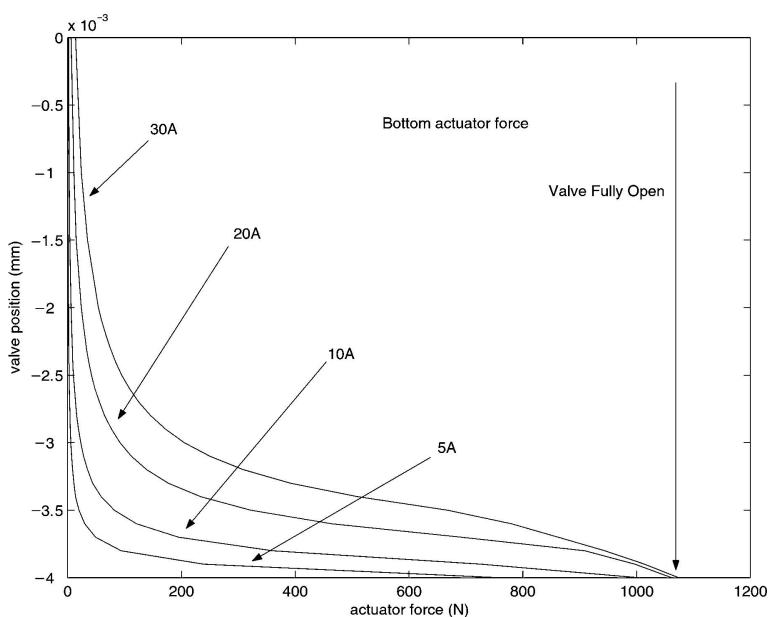

Fig. 10 Solenoid actuator static force-current-displacement characteristic 


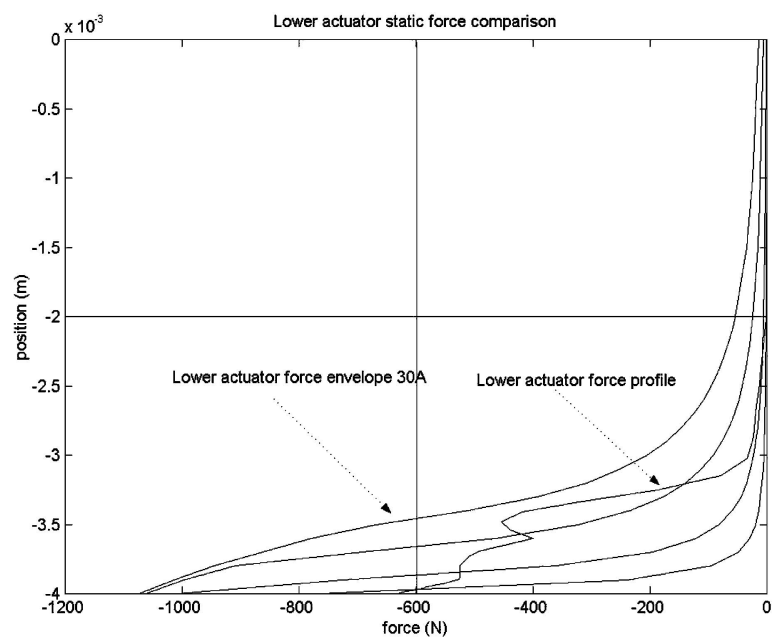

Fig. 11 Comparison of the solenoid actuator static force-current-displacement characteristic with optimal force trajectory (lower)

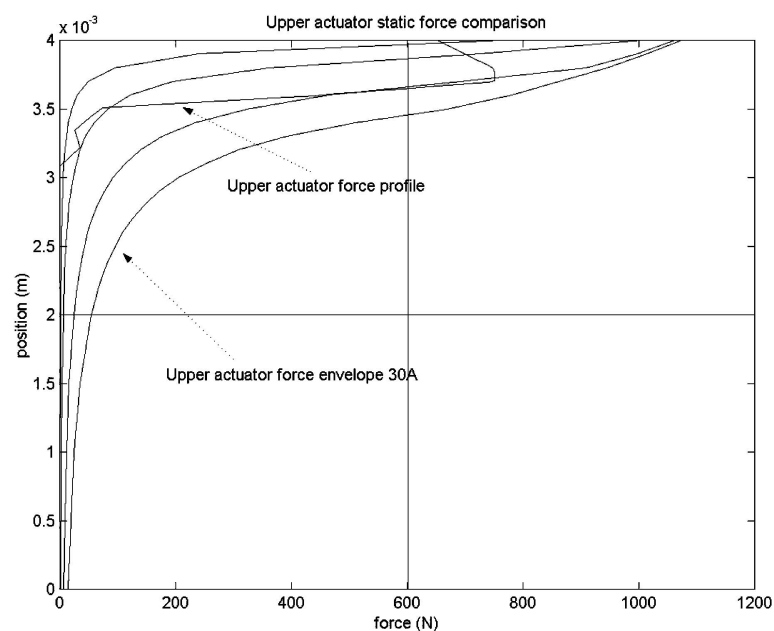

Fig. 12 Comparison of the solenoid actuator static force characteristic with optimal force trajectory (lower)

operational terms cannot be confirmed. An electromagnetic dynamic model approximation is developed to allow multiobjective analysis again to be applied.

This time the analysis will seek to confirm the controllability of the actuator, that is, whether a control action trajectory exists that satisfies all the dynamic requirements and constraints. In an iterative environment, further design steps take place if the design cannot satisfy the project requirements. The relationships between flux, current, and force ascertained by finite element analysis were studied (Figs 13 and 14) and were implemented as a Simulink model (Fig. 15). The electromagnetic model is coupled to the mechanical model and provides a platform on which to postulate the question 'is this system controllable in the sense of performance requirements and design

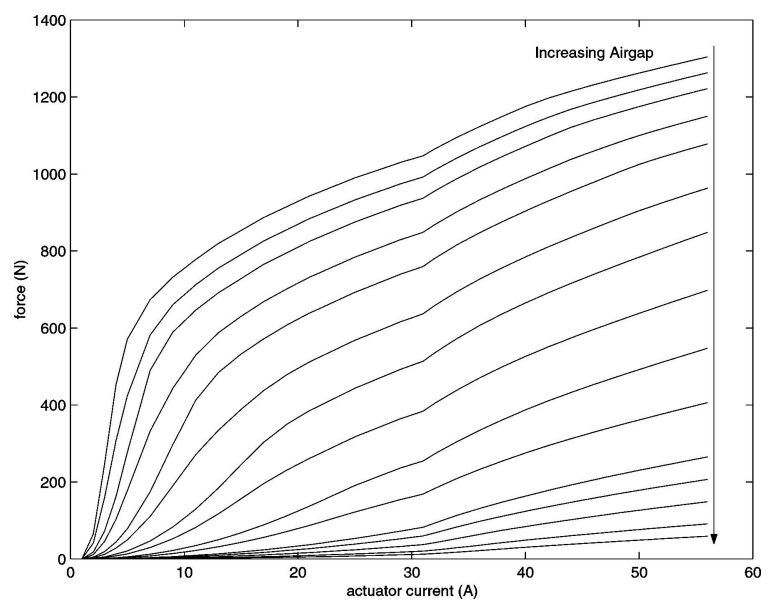

Fig. 13 Actuator force-current-displacement map from finite element analysis

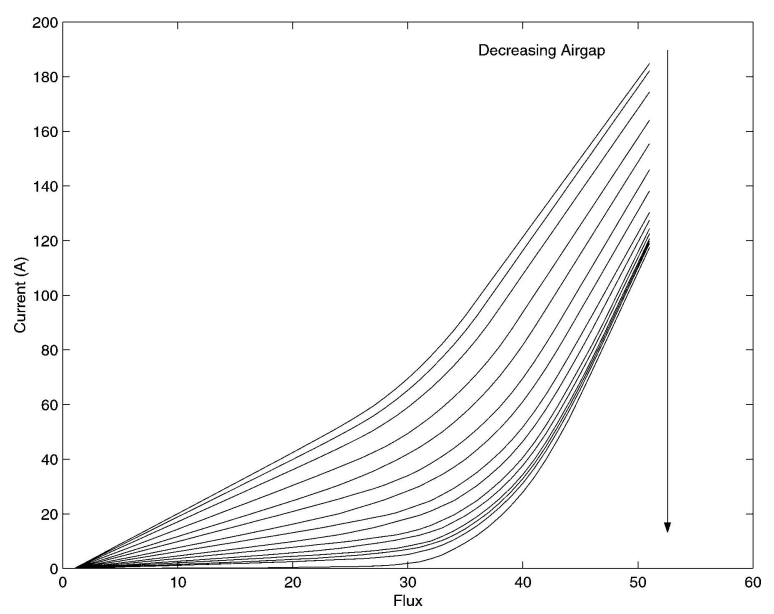

Fig. 14 Actuator flux-current-displacement map from finite element analysis

constraints?' The system is controllable in this sense if an applied current trajectory exists that, when applied to the actuators, causes the armature to move in a position-velocity path that satisfies the mechanical constraints of the application (soft landing, etc.). The current trajectory must also comply with the maximum current constraint, and voltage must be limited to the application d.c. link voltage. To achieve this end, a multiobjective analysis is constructed with the same quantization of decision variables as before. In this case, the decision variables provide a position-current demand vector to be tracked by the electromechanical model (Fig. 15). Tracking is achieved by a PID current controller, with the multiobjective evolutionary algorithm set to the same operational parameters and objective function as before (with 'force' replaced by 'current'). Also, the number of objectives was reduced to 6 by omitting 


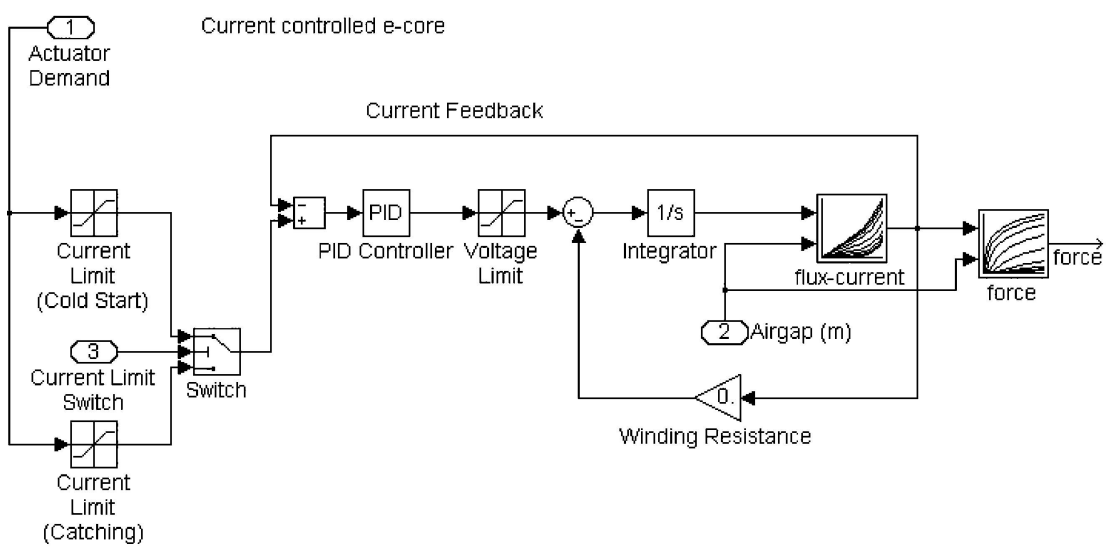

Fig. 15 Electromagnetic model for controllability analysis

the overall maximum current objective to reduce the computation time, since it had been found that the force-distance penalties performed the same function.

\section{RESULTS}

Figure 16 is the non-dominated trade-off output from the multiobjective analysis. The objectives to be minimized are numbered as follows.

1. Actuator transition time.

2. Bottom actuator distance $e^{2} \times$ current.

3. Soft landing velocity.

4. Current derivative.

5. Soft release (valve gap closing) velocity.

6. Top actuator distance ${ }^{2} \times$ current.

It is immediately apparent that the controllability of the system is dependent on conflicting objectives,

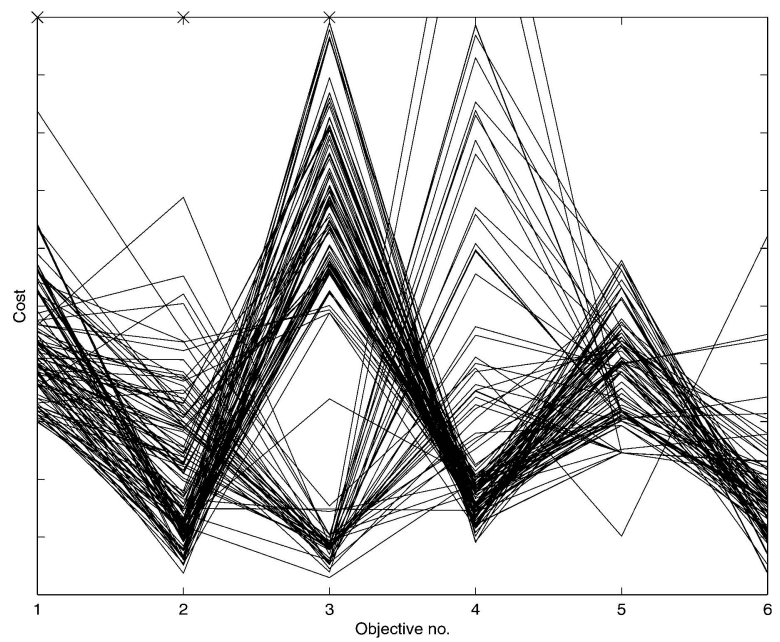

Fig. 16 Non-dominated trade-off graph for the actuator controllability analysis revealing the salient operating principles of the constrained system. The following examples can be given.

1. The actuator transition time (1) can be reduced by relaxing the constraints on currents applied at large air gaps (2), but this incurs a cost in terms of the soft landing (3).

2. Soft landing (3) and soft release (5) incur a cost in terms of the current derivatives (4).

3. Close examination of the trade-off graph reveals a particularly concerning trade-off, that is, there are only a small number of candidate solutions in which both soft release and soft landing are achieved.

The single successful solution selected does comply with all the required dynamic project requirements in terms of soft landing, soft departure, and transition time (Figs 17 to 19). The constraint of $40 \mathrm{~A}$ maximum current was, however, found in all cases impossible to comply with to achieve the required dynamic performance. Although the $42 \mathrm{~V}$ supply

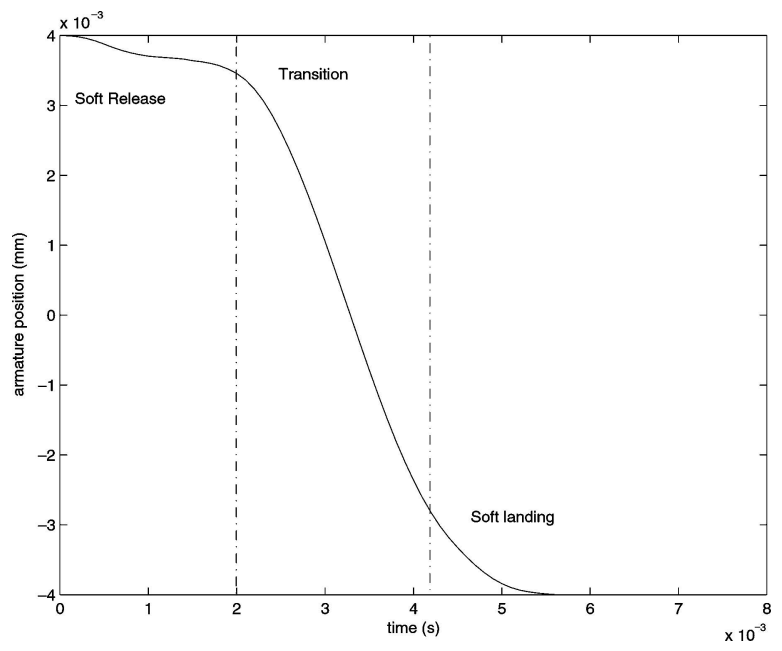

Fig. 17 Successful time-position trajectory 


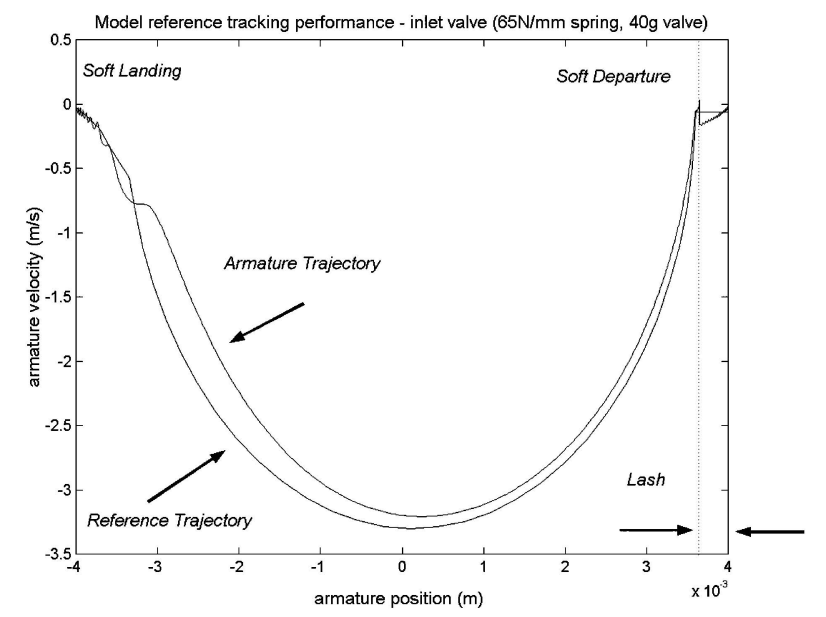

Fig. 18 Reference/tracking performance of successful controller
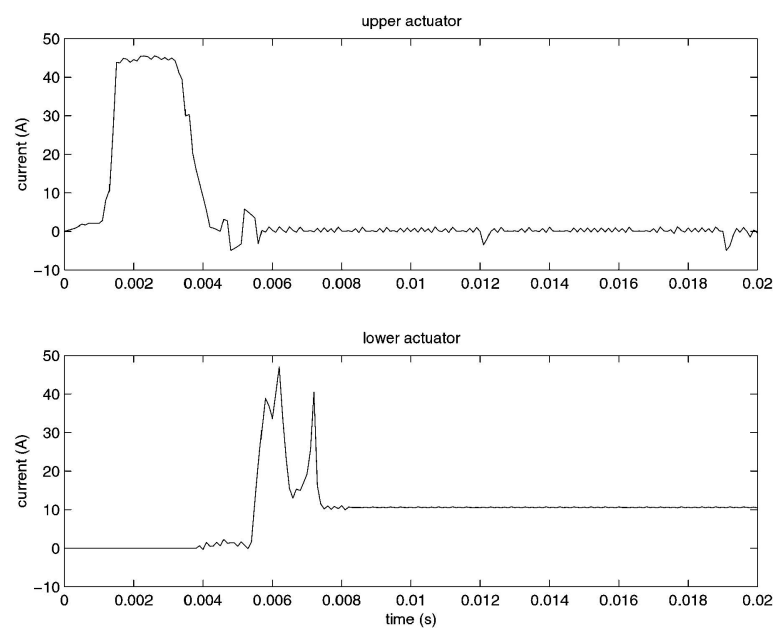

Fig. 19 Upper and lower actuator current waveforms voltage was strictly adhered to, it was found that the maximum current constraint needed to be relaxed in order to achieve the performance requirements. With a current limit of $45 \mathrm{~A}$, a current trajectory does exist, which when tracked by standard PID current control loops produces a position-velocity trajectory for the armature that complies with the required dynamic performance. A revised current limit has also been identified that allows these objectives to be achieved. The approach has identified a means of confirming the controllability of complex non-linear systems.

\section{CONCLUSIONS}

An application example has been presented in order to illustrate a technique for answering the question as to the controllability of the system. Although the example investigates an existing actuator candidate, it is envisaged that the technique will exist as part of a greater multiobjective design process that includes actuator redesign (Figs 20 and 21). It has been shown that, in the example, the actuator as designed does satisfy the static force requirements and thus should be capable of effecting adequate operation of the armature. However, in practice the control design to achieve all the project performance objectives was proving difficult to achieve. In general the multiobjective controllability analysis would have been performed before the manufacture of experimental actuators, and an iterative process performed with finite element analysis to achieve a controllable system. In the case presented here, it is possible to analyse the existing design and enter an iterative design phase to produce an improved actuator. It can

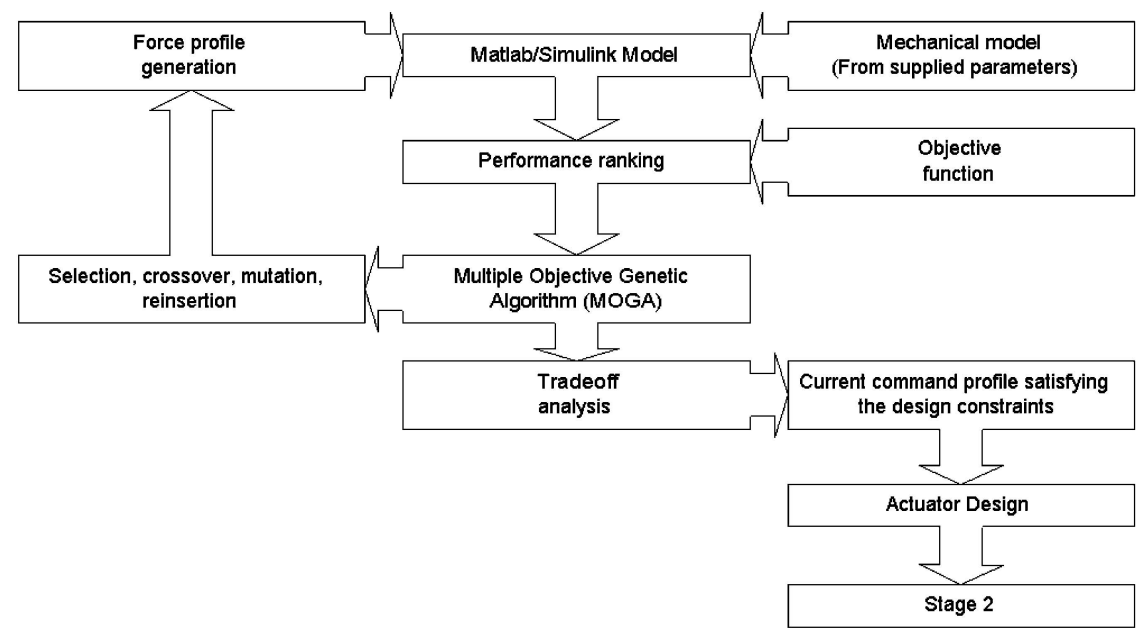

Fig. 20 Stage 1 analysis and design 


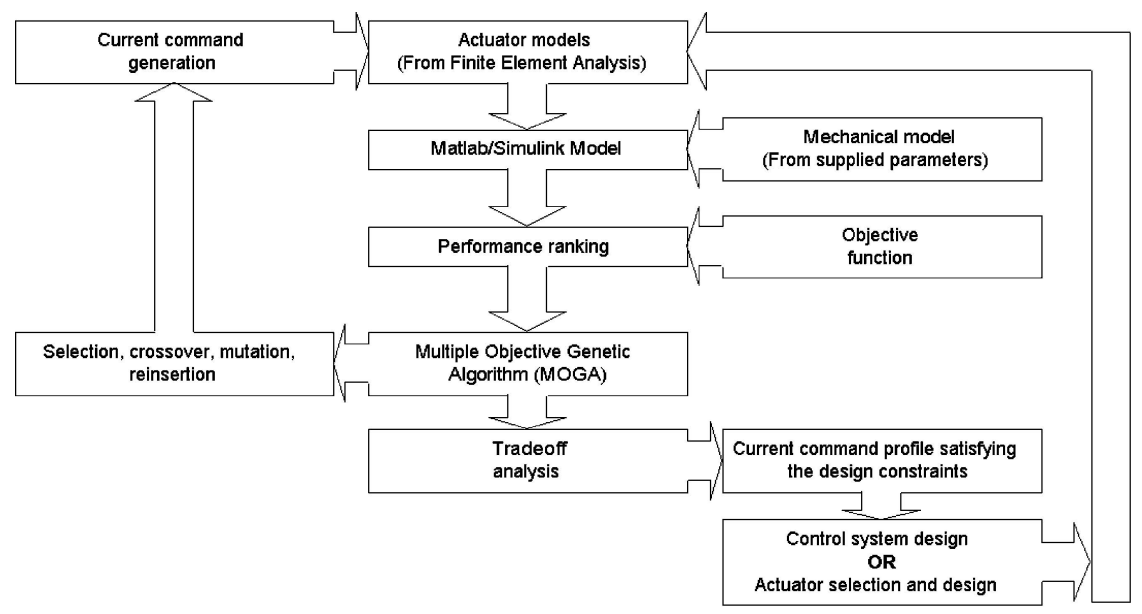

Fig. 21 Stage 2 analysis and design

also be seen that the technique has potential benefits to a wide range of applications where controllability analysis proves difficult or impossible by conventional methods.

It has been shown that the analysis has identified the requirement to increase the operational current limits in order to achieve controllability. It has also been shown that the group of successful candidate current vector solutions is extremely small. This is an area of the technique deserving further research. It would certainly verify the experimental problems that have been experienced implementing successful control design on the system. A conclusion that appears to be valid would be that the multivariable controllability analysis of the system also reveals a subset of potential robustness in any designed control systems. The small number of candidate solutions shows that the family of current vectors that can achieve controllability is small, and indeed narrow. This is confirmed by experimental experience which suggests that the system is extremely susceptible to physical parameter variations.

The findings would suggest that the system is indeed controllable, but further iterations of the design procedure are necessary. This conclusion stems from the narrow band of successful solutions, suggesting an extremely sensitive system, and, coupled to this, the relationship between the current demand derivatives and soft release/landing. It was found in the search for successful candidate solutions that the restrictions on the current demand vector derivatives needed to be relaxed to achieve controllability, suggesting a significant limitation to controllability. After a narrow successful band, further relaxation produces no further successful candidates. This suggests that the time constants of current and flux (and hence force production) are limiting factors at small air gaps, and thus the system would benefit in both controllability and robustness from further iterative design steps in terms of the actuator design. This process is currently in progress.

The process has successfully identified limitations and potential solutions to achieving controllability of a non-linear constrained multiobjective system. Although controllability has been confirmed, the method has also confirmed experimental experience regarding the difficulty of implementing successful control design. Although the technique is relatively new, its potential benefits are apparent for developing new systems, as is the potential for identifying controllability issues with existing systems.

\section{ACKNOWLEDGEMENTS}

The authors would like to thank Dr Richard Clark of the Electrical Machines and Drives group at Sheffield University, UK, for providing the finite element analysis of the electromagnetic actuator.

\section{REFERENCES}

1 Park, S.-H., Lee, J., Yoo, J., Kim, D., and Park, K. Effects of design and operating parameters on the static and dynamic performance of an electromagnetic valve actuator. Proc. Instn Mech. Engrs, Part D: J. Automobile Engineering, 2003, 217, 193-201.

2 Wang, Y., Stefanopoulou, A., Haghgooie, M., Kolmanovsky, I., and Hammoud, M. Modelling of an electromechanical valve actuator for a camless engine. 5th International Conference on Advanced Vehicle Control, University of Michigan, USA.

3 Hermann, R. and Krener, A. J. Nonlinear controllability and observability. IEEE Trans. Autom. Control, 1977, (22), 728-740. 
4 Nijmeijer, H. Controllability distributions for nonlinear systems. Syst. Control Lett., 1982, (2), 122-129.

5 Van der Schaft, A. J. Observability and controllability for smooth nonlinear systems. SIAM J. Control Optimisation, 1982, 20, 338.

6 Chipperfield, A. and Fleming, P. J. Multiobjective gas turbine engine controller design using genetic algorithms. IEEE Trans. Ind. Electronics, 1996, 43(5), $1-5$.

7 Fonseca, C. M. and Fleming, P. J. Multiobjective optimisation and multiple constraint handling with evolutionary algorithms. Part 1: a unified formulation; Part 2: application example. IEEE Trans. Syst., Man and Cybernetics, Part A: Syst. and Humans, 1998, 28(1), 26-37, 38-47.

8 Zaoui, F. and Marchand, C. Using genetic algorithm for the optimization of electromagnetic devices. COMPEL - Int. J. for Comput. and Math. in Elect. and Electronic Engng, 1998, 17(1-3), 181-185.

9 Sadiku, M. N. O. Elements of electromagnetics, 2nd edition, 1989, pp. 382-383 (Oxford University Press, Oxford).

10 Purshouse, R. C. and Fleming, P. J. The multiobjective genetic algorithm applied to benchmark problems - an analysis. Research report 796, Department of Automatic Control and Systems Engineering, University of Sheffield, UK, August 2001.

11 Fonseca, C. M. and Fleming, P. J. Genetic algorithms for multiobjective optimisation: formulation, discussion and generalisation. In Genetic algorithms: proceedings of the fifth international conference, 1993, pp. 416-423 (Morgan Kaufmann, San Mateo, California).

12 Goldberg, D. E. Genetic algorithms in search, optimisation and machine learning, 1989 (AddisonWesley, Boston).

13 Schroder, P., Green, B., Grum, N., and Fleming, P. J. On-line evolution of robust control systems: an industrial active magnetic bearing application. IFAC J. Control Engng Pract., 2001, 9(1), 37-49.

14 Griffin, I. A., Schroder, P., Chipperfield, A. J., and Fleming P. J. Multiobjective optimisation approach to the ALSTOM gasifier problem. Proc. Instn Mech. Engrs, Part I: J. Systems and Control Engineering, 2000, 214, 453-468.
15 Simkin, J. and Trowbridge, C. W. Optimization problems in electromagnetics. IEEE Trans. Magnetics, 1991, 27, 4016-4019.

16 Ratnajeevan, S. and Hoole, H. Computer aided analysis and design of electromagnetic devices, 1989 (Elsevier, New York).

17 Zitzler, E. and Thiele, L. Multiobjective evolutionary algorithms: a comparative case study and the strength Pareto approach. IEEE Trans. Evolutionary Comput., 1999, 3(4), 257-271.

\section{APPENDIX}

\section{MOGA parameters}

Population size: 100.

Number of decision variables: 27.

Number of objectives: 7 .

Number of immigrants per generation: 6 .

Coding: grey, 20 bits per decision variable, except where varied [17].

Selection: stochastic universal sampling.

Recombination: single-point binary crossover, probability 0.7 .

Mutation: element-wise bit flipping, expectation of 1 bit per chromosome.

Generational gap: zero.

Random injection: two random chromosomes per generation.

Elitism: none.

Fitness assignment: Fonseca and Fleming [11] multiobjective ranking. Transformation from rank to fitness using linear fitness assignment with rank-wise averaging.

External population: off-line storage of nondominated solutions.

Fitness sharing: (parameter-less) Epanechnikov fitness sharing [7] implemented in criterion space.

Mating restriction implemented: distance set to the niche size parameter found by the Epanechnikov sharing algorithm. 